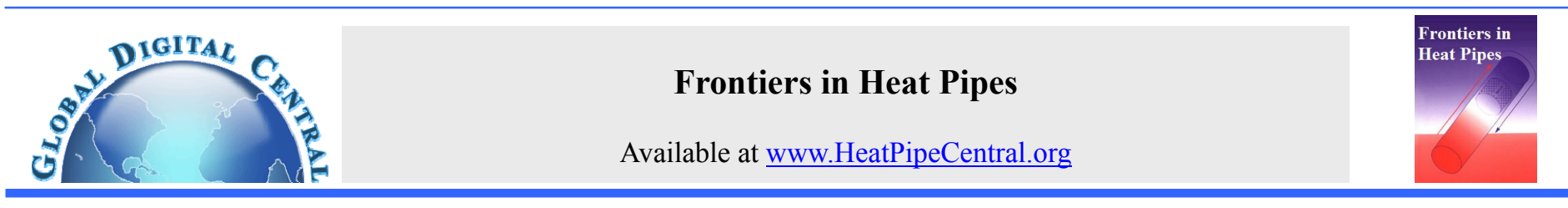

\title{
HYDRODYNAMICS OF A CONFINED MENISCUS IN A SQUARE CAPILLARY TUBE AT LOW CAPILLARY NUMBERS
}

\author{
Gyana Ranjan Rana, Basant Singh Sikarwar, Sameer Khandekar, P. K. Panigrahi \\ Department of Mechanical Engineering, Indian Institute of Technology Kanpur, Kanpur 208016 India
}

\begin{abstract}
In microchannel fluid flow, understanding of the liquid-gas interface behavior is vital for developing a wide range of microfluidic devices. The dynamic contact angle of the liquid-air meniscus varies with its velocity and the ensuing meniscus shape has profound effect on the local transport characteristics in its vicinity. Depending on the application, dynamic menisci shapes eventually control the momentum, heat and mass transport coefficients in two-phase microchannel flow geometries, where such conditions are often encountered. To better understand the effect of dynamic contact angle on meniscus shape, high speed visualization of menisci of four different liquids (water, ethanol, glycerin and silicon oil) has been undertaken at different Capillary numbers. Quantitative information of the velocity field and its distribution near the moving liquid-air interface has been done using micro-PIV measurements in a $1 \mathrm{~mm} \times 1 \mathrm{~mm}$ dry square capillary having deionized water as the working fluid. This provides vital information on the local flow transport characteristics (two-dimensional velocity fields on a longitudinal plane) in the wake of the meniscus. To augment and complement the study, three-dimensional simulation of the flow field near the liquid-air meniscus has also been performed on Comsol ${ }^{\circledR}$, applying the two-phase flow level-set method. The results clearly demonstrate that, while the u-velocity profile in the liquid domain is parabolic (Poiseuille-type flow) in nature away from the interface, it drastically changes (become flatter) as we approach the meniscus. Close to the meniscus the flow becomes three-dimensional with both $\mathrm{v}$ and $\mathrm{w}$ velocities showing a double-vortex, the strength of the latter being lower than the former. This observation is clearly noted both by PIV data as well as the simulations. The characteristic of the flow field in the meniscus wake is the most important parameter which affects the viscous stress generated due to the meniscus motion. The study reveals that controlling the wettability of the liquid can be an effective tool to control the overall transport behavior of the moving confined meniscus.
\end{abstract}

Keywords: Micro-PIV experiments, Meniscus shape, Capillary number, Interface dynamics, Square capillary, Flow simulation

\section{INTRODUCTION}

Transport phenomenon at liquid-air interface inside microscale devices has emerged as an important research field due to its relevance in many upcoming systems and devices. For example, interfacial flows occur in micro-total analysis system (Juncker et al., 2002), in lab-on-a-chip devices (Walker and Beebe, 2002), micro fuel cells (Pattekar and Kothare, 2004) and micro-/pulsating heat pipes (Khandekar et al., 2010). From a transport perspective, creation of two-phase fluid flows (gas-liquid, vapor-liquid or liquid-liquid systems) inside micro-channel geometries is considered to be one of the most suitable techniques available for enhancement of heat/mass transfer. Transportation of small fluid volume inventory with the advantage of large available interfacial area and small diffusion paths, all lead to attractive advantages from such systems. Understanding the fundamental hydrodynamics of such flows inside confined microchannel geometries is helpful for enhancing the performance parameters in applications like high speed coating, dying, adhesive printing, flow through porous media (petroleum industry), passive heat pipes, laser devices, bio-fluid engineering and bio-medical diagnostics and in chemical and material synthesis etc. Systematic physical insight into the local fluid flow and transport characteristics of dynamic liquid-air interfaces is thus vital for developing a wide range of such emerging micro-devices and systems.
While many of the reported experimental studies have been carried out using circular capillary tubes, interfacial flows inside a square capillary is more complex due of the difficulty in general treatment of motion (Thulasidas et al., 1997; Ajaev and Homsy, 2006). For many applications involving array of microchannels on flat plate substrates, a square or trapezoidal cross sectional geometry is preferred due to manufacturing routes employed. Furthermore, from a fundamental internal convective flow consideration, all other conditions remaining identical, a square channel provides lesser pressure drop than an equivalent circular channel (Shah and London, 1978).

Till now, a variety of experimental and numerical techniques have been adopted to analyze the flow field inside microchannels (Torralba et al., 2005). One popular contemporary technique is the micro-Particle Image Velocimetry ( $\mu$-PIV), which offers a powerful, nearly nonintrusive tool to study the liquid flow field. Santiago et al. (1998) developed a micron-resolution $\mu$-PIV to measure ensemble-average and instantaneous liquid flow field in micro-scale devices. They measured velocities in a Hele-Shaw flow around a $30 \mu \mathrm{m}$ elliptical cylinder, with a bulk velocity of $50 \mu \mathrm{m} / \mathrm{s}$ using an epi-fluorescent microscope and 100 $300 \mathrm{~nm}$ diameter seeding particles. They also specified that the spatial resolution and accuracy of fluid velocity measurements is limited by

* Corresponding author: samkhan@iitk.ac.in 
the diffraction limit of the recording optics, noise in the particle image field, the interaction of fluid with finite size seed particles and Brownian motion. Meinhart et al. (1999) have presented the ensemble averaged velocity measurement in a $30 \mu \mathrm{m}$ x $300 \mu \mathrm{m}$ rectangular microchannel. Measurements were obtained using low spatial resolution away from the wall and a higher spatial resolution close to the wall. Reported PIV measurements were within $2 \%$ of the analytical solution for the flow inside a rectangular channel. Lima et al. (2007) also obtained nearly parabolic velocity profiles for pure water and invitro blood, flowing through a $100 \mu \mathrm{m}$ square-microchannel at low Reynolds number. Gunther et al. (2004) used $\mu$ PIV to characterize the segmented liquid-gas flow at low Capillary number. The recirculation patterns inside the liquid slug and the symmetric nature of this recirculation was reported. It was pointed that the surface roughness and the compressibility of gas phase are the main reasons behind the loss of symmetry for the velocity inside liquid slugs. This enhances mixing across the centerline of straight micro-channels. Li and Olsen (2006) used micro-PIV to measure instantaneous velocity profiles in a square Poly-di-methyl-siloxane (PDMS) micro-channel with hydraulic diameter ranging from $200 \mu \mathrm{m}$ to $640 \mu \mathrm{m}$ and for Reynolds number ranging from 200 to 3971 , having water as the working fluid. They calculated mean velocity profiles, velocity fluctuations, Reynolds stresses and spatial correlations of velocity fluctuations. Silva et al. (2008), using micro-PIV technique, observed the effect of the wall surface roughness on velocity profiles inside micro-capillary tubes and compared their results with numerical CFD, without taking surface roughness into account. They found that not considering the surface roughness leads to underestimation of friction factor by about $11 \%$. Kinoshita et al. (2007) used confocal micro-PIV system to measure and investigate the internal flow of a droplet, transported in a square PDMS microchannel having a size of $100 \mu \mathrm{m} \times 58 \mu \mathrm{m}$. They showed that the droplets have three-dimensional flow structures. This originates due to the drag force on the contact surfaces with the surrounding walls of the channel when the droplet moves inside a square microchannel.

Most of the experiments have been carried out to study the velocity profiles, either far away from the moving liquid-air interface or, alternately, bulk velocity profiles inside liquid plugs using microPIV (Meinhart et al., 1999; King et al., 2007, Malsch et al. 2008; Zimmerman, 2010) have been reported. These studies generally indicate that the liquid velocity profile is parabolic, satisfying the noslip boundary condition at the wall. However, the flow phenomenon very close to the liquid-air interface has not been clear due to its complexity and three-dimensional nature. While numerical and analytical work has been reported (for example, by Chebbi, 2003; Egorov et al., 2003; He et al., 2010; Mehdizadeh et al., 2011), extensive experimental data on near meniscus flow fields is not easily available.

For micro-fluidics, the effect of surface forces becomes prominent during two-phase flow which may also result in the formation of threephase contact lines with the tube/duct wall. Thus, the surface interaction refers not only to the interfacial interactions between two fluid phases, but also to the interaction of each fluid with channel walls (Huh et al. 2009). Wang et al. (2013) found that the surface wettability of micro-channel walls profoundly influenced both, the nature of twophase flow and the flow regime transition. Lee and Lee (2008a) studied the pressure drop of two-phase plug flows in round small channels with diameters ranging from 1.62 to $2.16 \mathrm{~mm}$ and for tube materials of different wetting properties. They concluded that the pressure drop in small channels was significantly affected by the wetting condition of channel wall, but the effect of wettability was not introduced in their prediction model for pressure drop profile. Yamada et al. (2011) found that the product of friction factor and Reynolds number for hydrophobic channels increased as Reynolds number increased in the laminar flow regime. Bajpai and Khandekar (2013) reported with the help of three-dimensional simulations that the variation of dynamic contact angle of a confined meniscus moving inside a capillary tube affects the local heat transfer coefficient close to three-phase triple line.
When liquid plug flows in a small diameter tube $($ Bo $<2)$, the capillary forces play a major role and gravity becomes relatively insignificant. In such a case, the interfacial tension between solid-liquid $\left(\sigma_{s l}\right)$ and solid-gas $\left(\sigma_{s g}\right)$ should also be taken into account during flow analysis. Considering an isolated moving liquid plug inside a capillary tube which is enclosed by two menisci, the dynamic contact angle formed between the flowing liquid meniscus and the solid surface is achieved by a balance between the capillary and the viscous forces (Berthier, 2008). Thus, the moving menisci of the liquid plug are characterized by dynamic advancing and receding contact angles, which are different from the static equilibrium contact angle, because of the interplay between surface tension and viscous forces. The relative scaling of these two forces is represented by the Capillary number $(\mathrm{Ca}=\mu U / \sigma)$. Rose and Heins (1962) were among the first who highlighted the relation between the advancing contact angle and average velocity $U_{a v g}$ of three-phase contact line. Later, various researchers like Hoffman (1975), Tanner (1979) and Blake (2006) studied the wetting behavior and dependency of the advancing contact angle on $\mathrm{Ca}$ and the static contact angle.

Advent of microfluidic devices has directed considerable research attention to interfacial dynamics and transport phenomena at small scale devices. The complex flow phenomenon close to the liquid-air interface in a moving confined meniscus needs further attention and clarity. Heat and mass transfer rates are greatly affected by the meniscus shape, which, in turn is a function of local flow dynamics. The three-dimensionality of the flow in close proximity of the meniscus needs to be understood for proper design equations for the viscocapillary dissipation and resulting local transport. In this background, $\mu$-PIV study has been carried in the liquid domain (D.I. water) of a moving liquid-air meniscus inside a dry square glass capillary tube of size $1 \mathrm{~mm} \times 1 \mathrm{~mm}$. As the liquid enters the dry capillary from one end with a constant velocity (i.e., fixed $\mathrm{Ca}$ ), it creates a confined liquid-air meniscus inside it. It must be noted that the dynamic contact angle is a function of the Capillary number of the meniscus and thus, depending on the flow condition, which includes the effect of wettability, the shape of the meniscus changes, affecting the flow field close to it. This has been scrutinized by high speed visualization in conjunction with the PIV flow field measurements, to understand the mechanism of enhancement of local and average transport coefficient. The numerical difference between the incompressible three-dimensional continuity equation and the summation of the gradients of $u$ and $v$ velocity obtained by PIV (i.e., $(\partial u / \partial x+\partial v / \partial y)$, indicates the three-dimensional nature of the near-meniscus flow field.

To augment and complement the present study, three-dimensional numerical flow field simulations near the liquid-air meniscus have also been carried out on commercial finite element solver, i.e., Comsol ${ }^{\circledR}$. Wettability of the liquid and the flow Capillary number are the required parameters of interest. The experimental flow fields close to the moving liquid-air interface obtained from PIV measurements are compared with the simulations. After benchmarking of the data, the wettability parameter is varied in the simulations to highlight its isolated and explicit effect on local flow transport characteristics. The results clearly demonstrate that, very close to the interface, the $u$ velocity profile is not parabolic in nature (becomes flatter), while away from the interface (going in the liquid domain) it satisfies the Poiseuille flow condition. Close to the meniscus, the flow clearly becomes threedimensional with both $v$ and $w$ velocities showing a double-vortex, the strength of the latter being an order-of-magnitude lower than the former. This three-dimensionality leads to enhanced local transport of momentum. The simulations also reveal that controlling the wettability of the liquid can be an effective tool to control the viscous shear and therefore the pressure drop required to move the meniscus; the meniscus region of liquids with comparatively higher wettability manifests a stronger shear stress as compared to a non-wetting liquid meniscus moving at the same Capillary number. 


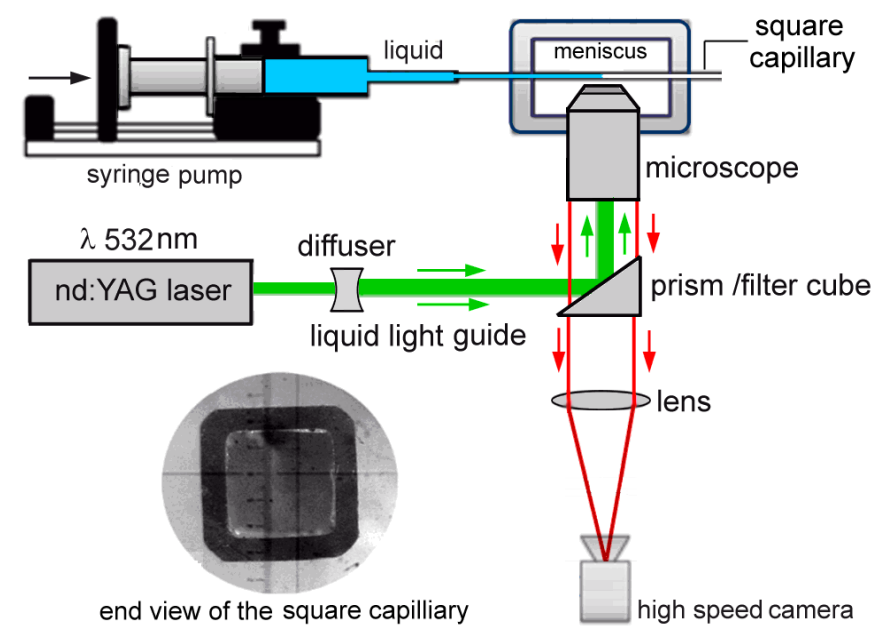

Fig. 1 Schematic diagram of the videography and $\mu$-PIV setup for fluid flow velocity measurement inside glass capillary tubes of size $1 \mathrm{~mm} \times 1 \mathrm{~mm}$.

\section{EXPERIMENTAL DESCRIPTION}

The schematic of experimental setup for high speed video imaging (by Photron-Fastcam-SA $3^{\circledR}$ ) and flow field measurement by micro-PIV (Dantec Dynamics ${ }^{\circledR}$ ) close to the moving liquid-air meniscus is shown in Figure 1. It consists of a syringe infusion pump (Cole Parmer\#74900-00) for supply of the working fluid, the output of which is connected to the horizontally placed square capillary tube of cross sectional size $1 \mathrm{~mm} \times 1 \mathrm{~mm}$. Before starting each new run of the experiment, the glass capillary is thoroughly cleaned in an ultrasonic bath by a standard protocol and completely dried in an oven maintained at $120^{\circ} \mathrm{C}$. The working fluid is supplied at a constant feed rate to the capillary tube; the liquid-air meniscus thus formed travels at a fixed Capillary number inside the (already dry) capillary tube. The liquid-air meniscus visualization has been performed for four different liquids: de-ionized water, pure ethanol, silicon oil and glycerin. While qualitative visual data is reported here, the detailed numerical data of advancing apparent contact angle with the Capillary number is being reported elsewhere. The arrangement of Photron-Fastcam-SA3 camera for visualization of variation of the advancing contact angle with respect to the given Capillary number is shown in Figure 1.

Micro-PIV experiments were performed with deionized water, both under bright field mode and fluorescent mode (both results compared well with each other, however, for lack of space, only bright field data will be reported here). The dry glass square capillary was placed horizontally over the stage of the inverted microscope $(5 \mathrm{X}$ objectives) of the micro-PIV unit (Leica ${ }^{\circledR}$ Micro-system; DMI 5000). The maximum operating frequency of this micro-PIV was $6 \mathrm{~Hz}$. The working fluid (de-ionized water), having sufficient seeding particles (glass particles of size $10 \mu \mathrm{m}$ in concentration $12.5 \mathrm{mg} / \mathrm{ml}$ of liquid for bright field imaging and fluorescent carboxylate particles of size $1 \mu \mathrm{m}$ in concentration $1 \mu \mathrm{l} / \mathrm{ml}$ of liquid for florescent mode imaging), was supplied to the square capillary at specified calibrated flow rate. Successive images of the region of interest, just behind the dynamic liquid-air meniscus interface, were taken, using a 12 bit, $1344 \times 1024$ pixels digital CCD Camera (geometric resolution 1 pixel $=0.75$ microns), for PIV flow analysis. All the measurements were taken at the mid plane of the meniscus, a plane which was $0.5 \mathrm{~mm}$ up from the bottom wall (relate this to Figure 2). Proper masking of the acquired PIV image was carried out by carefully tracking the seeding particles movement near the wall and at the interface to remove the non-flow outside region from the captured image field. The micro-PIV system produced two successive images and an adaptive cross correlation algorithm was applied to estimate the instantaneous flow field. An interrogation area of $64 \times 64$ pixels was chosen with a $50 \%$ overlap both horizontally and vertically. For removing unreasonable velocity vectors, appropriate filtration was applied after masking the spurious velocity vectors. The acquired PIV images were processed using Dantec ${ }^{\circledR}$ Dynamic software to obtain the velocity at each point in the flow field in the liquid domain.

The micro-PIV analysis was done for two different average water flow rates, $0.6 \mathrm{ml} / \mathrm{hr}$ and $1.0 \mathrm{ml} / \mathrm{hr}$ (corresponding to $U_{\text {avg }}=0.166 \mathrm{~mm} / \mathrm{s}$ and $0.277 \mathrm{~mm} / \mathrm{s}$ ), the higher flow rate being limited by the upper frequency response of the micro-PIV system. Velocity profiles across the channel width, at a fixed $z$-plane (as shown in Figure 2), were plotted for different stream wise locations just near and behind the dynamic liquid-air meniscus. For validation and benchmarking of the data away from the interface, the following analytical solution for fully developed velocity profile inside a square channel (Eq. 1), as given by Bruus (2008), has been used,

$$
u(y, z)=\frac{48 Q}{\pi^{3} a b} \frac{\sum_{n, \text { odd }}^{\infty} \frac{1}{n^{3}}\left[1-\frac{\cosh (n \pi y / a)}{\cosh (n \pi b / 2 a)}\right] \sin (n \pi z / a)}{1-\sum_{n, o d d}^{\infty} \frac{192 a}{n^{5} \pi^{5} b} \tanh (n \pi b / 2 a)}
$$

where, $a$ and $b$ are the height and width of the rectangular channel, respectively, which in the present case of a square channel are identical.

The micro-PIV measurement data provides two components of velocity, i.e. $u$ and $v$ in the directions $x$ and $y$, respectively, on a definite plane of observation. In all regions where the flow remains essentially two-dimensional in nature, the 2-D continuity equation for the flow field, i.e., $(\partial U / \partial x)+(\partial V / \partial y)=0$, must be satisfied. If there remains a non-zero residual of the two-dimensional continuity equation, it is a definitive indication that the flow field is no more two-dimensional but is indeed three-dimensional, wherein the residual points towards the gradient of the $w$ velocity in the z-direction. Thus, after obtaining the 2D velocity field ( $u$ and $v$ ) from the micro-PIV experiments, a fourpoint central difference method having a fourth order truncation error was applied at each grid point inside the domain to estimate the residual in the $2 \mathrm{D}$ continuity equation. The corner points of the domain were handled by either a forward difference or a backward difference scheme, as applicable. This methodology provides a simple plan for checking the fluid flow behavior and deviations, if any, from twodimensional nature.

\section{NUMERCAL FORMULATION AND SIMULATION METHODOLOGY}

Simulation of the isolated liquid-air meniscus traveling with constant velocity inside a square capillary tube has been done using the commercial platform COMSOL ${ }^{\circledR}$ (Comsol 4.3a documentation, 2012). The evolution of the gas-liquid interface, and associated flow in the liquid domain and the adjoining air domain, as the interface moves, has been simulated using the pseudo 'single-fluid' approach. While the flow field is shared by both the fluids, the interface is tracked by an additional advection equation. The hydrodynamics of such flows can then be described by the usual equations for the conservation of mass and momentum, coupled with the additional interface advection which assigns level ' 1 ' to the pure liquid domain and level ' 0 ' to the pure gas phase, the interface being described by range of values in between 0 and 1 [Tornberg et al., 2000; Olsson and Kreiss, 2005]. The thermophysical properties of the interface are also scaled using the level-set. For an incompressible, Newtonian fluid, these equations can be written in tensor notation as follows: 


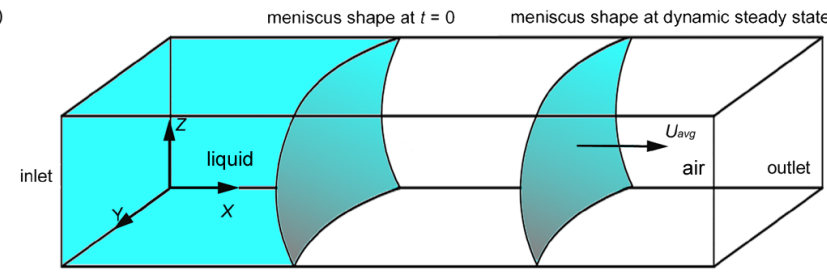

Computational domain

(b)

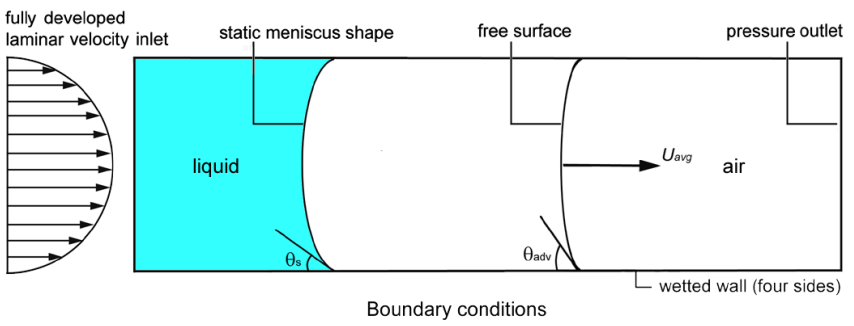

Fig. 2 (a): 3-D view of the computational domain of square capillary containing water and air (b) 2-D view with boundary conditions (at inlet: laminar velocity profile, at meniscus: interface boundary condition, at outlet: zero pressure condition and at wall: wetted no slip). The contact angle varies from static to dynamic at a given Capillary number as the meniscus moves from rest and attains stead-state motion.

Continuity equation:

$\frac{\partial \rho}{\partial t}+\frac{\partial \rho u_{j}}{\partial x_{j}}=0$

Momentum equation:

$\frac{\partial \rho u_{i}}{\partial t}+\frac{\partial \rho u_{j} u_{i}}{\partial x_{j}}=-\frac{\partial p}{\partial x_{i}}+\mu \frac{\partial u_{i}}{\partial^{2} x_{j}}+\left(F_{s}\right)_{i}+\left(F_{g}\right)_{i}$

The advection of the level-set function is described by:

$\frac{\partial \phi}{\partial \mathrm{t}}+\mathrm{u}_{\mathrm{i}} \frac{\partial \phi}{\partial x_{j}}=\gamma \frac{\partial}{\partial x_{j}}\left(\varepsilon \frac{\partial \phi}{\partial x_{j}}-\frac{\phi(1-\phi) \frac{\partial \phi}{\partial x_{j}}}{\left|\frac{\partial \phi}{\partial x_{j}}\right|}\right)$

where, $i$ is free indices and $j$ is the repeating indices. $u_{i}$ denotes the velocity vector in $i^{t h}$ direction, $p$ the pressure, $\rho$ the density and $\mu$ the dynamic viscosity of the fluid. $\left(F_{s}\right)_{i}$ is the surface tension force approximated as a body force in the vicinity of the interface, and $\phi$ is the level-set function of one of the phases. The bulk properties, such as density and viscosity, are determined from the volume-fraction weighted average of the properties of the two fluids. Hence, the density and viscosity are calculated from:

$\rho=\rho_{l}+\left(\rho_{l}-\rho_{\text {air }}\right) \phi$

$\mu=\mu_{l}+\left(\mu_{l}-\mu_{\text {air }}\right) \phi$

Figure 2 shows a schematic of the square micro-capillary and the appropriate boundary conditions applied. At the channel inlet, fullydeveloped laminar inflow conditions, as per Eq. (1), with prescribed volume flows are used. At the outflow boundary, the pressure is specified with no viscous stress; the latter is a reasonable assumption at low Capillary numbers. Initially, the liquid and air domain have to be specified along with any arbitrary shape of the interface. Once the static contact angle between the liquid and the channel wall is specified during initialization, the static shape of the interface is determined.
Subsequently, the simulation for the dynamic interface commences. The dynamic contact angle also needs to be specified as input condition, the estimation of which for a given $\mathrm{Ca}$ will be explained later. The singularity at the three phase contact line is avoided by taking a wetted wall boundary condition, wherein a slip length equal to the minimum grid size has been employed. The effect of gravity has been neglected as it plays a minor role as compared to the large surface tension forces due to microchannel geometry. An explicit geometric reconstruction scheme is used to represent the interface by using a piecewise-linear approach. The time-step used for the volume fraction equation (Eq. 4) in $\mathrm{Comsol}^{\circledR}$ is not necessarily the same as that used for the other equations (Eqs. 2 and 3) but is calculated based upon the characteristic transit time of a fluid element across a control volume and is limited by a specified maximum value for the Courant number (Co). The time taken to empty a cell is calculated by dividing the volume of each cell by the sum of the outgoing fluxes in the region near the fluid interface. The smallest such time is used as the characteristic time of transit for a fluid element across a control volume. The Courant number (Co) is defined by Eq. (7) as

$\frac{\Delta t}{\Delta x /\left(U_{\text {avg }}\right)_{l}}$

where, $\Delta x$ is the grid size and $\left(U_{a v g}\right)_{\text {fluid }}$ is the fluid velocity. A maximum Courant number of 0.001 is set in the present calculations and a variable time-step based on a fixed Courant number of 0.001 or less was used to force the time-step to be the same for all the equations.

Therefore, the terms on the left hand side (Eq. 4) give the correct motion of the interface, while those on the right hand side are necessary for numerical stability. The parameter $\varepsilon$, determines the thickness of the region where, $\phi$ goes smoothly from zero to one and is typically of the same order as the size of the element of the mesh. By default, $\varepsilon$ is constant within each domain and typically equals half the mesh size within the domain passed by the interface. The parameter $\gamma$ determines the amount of re-initialization and stabilization of level-set function. It needs to be tuned for each specific problem. If $\gamma$ is too small, the thickness of the interface might not remain constant, and oscillations in $\phi$ may appear because of numerical instabilities. On the other hand, if $\gamma$ is too large, the interface moves incorrectly. Recommended value of $\gamma$ scales with the magnitude of velocity of the specified problem.

The continuum surface force (CSF) model proposed by Brackbill et al. (1992) is used to model the surface tension effects. The CSF model approximates the surface tension induced-stress by a body force, which acts throughout a small but finite fluid region surrounding the interface. The surface tension force per unit area is represented as (Olsson and Kreiss, 2005):

$F_{s}=\sigma \kappa \delta\left(r-r_{\text {int }}\right) n$

where, $\sigma$ is the coefficient of surface tension, $\kappa$ is the radius of interface curvature, $n$ denotes the unit normal vector on the interface and $\delta(r)$ is the Dirac delta function, nonzero only at the fluid-air interface. The normal $n$ and curvature $\kappa$ in Eq. (8) are defined in terms of the level-set function $(\phi)$ via:

Table1: Properties of the gas and the liquid used in the simulations

\begin{tabular}{ccccc}
\hline Fluid & $\begin{array}{c}\text { Density } \\
\left(\mathrm{kg} / \mathrm{m}^{3}\right)\end{array}$ & $\begin{array}{c}\text { Viscosity } \\
(\mathrm{kg} / \mathrm{m} \mathrm{s})\end{array}$ & $\begin{array}{c}\text { Surface } \\
\text { tension } \\
(\mathrm{N} / \mathrm{m})\end{array}$ & $\begin{array}{c}\text { Static contact } \\
\text { angle } \theta_{s}(\mathrm{deg})\end{array}$ \\
\cline { 1 - 4 } Water & 998 & $8.899 \times 10^{-4}$ & 0.0732 & \\
\cline { 1 - 3 } Air & 1.175 & $1.751 \times 10^{-4}$ & - & 101.5 \\
\hline
\end{tabular}


$n=\frac{\nabla \phi}{|\nabla \phi|}$

and

$\kappa=\nabla n$

The level-set parameter $(\phi)$ is also used to approximate the delta function by a smooth function defined by (Sussman et al., 1994; Sussman et al., 1998):

$\delta=6(|\phi(1-\phi)|)(|\nabla \phi|)$

This implementation of the surface tension force can induce unphysical velocity near the interface known as spurious currents. This occurs because the pressure and viscous force terms do not exactly balance the surface tension force. The smaller time step and smaller size grid can help in minimizing these spurious currents. A second order, noniterative fractional step scheme is used for the time marching of the momentum and continuity equations. The residual tolerance for both the equations was set to $10^{-6}$.

The simulation is carried out in several steps. At first, the liquidair interface shape for the specified static contact angle of $\theta_{s}$ at the wetted wall of capillary tube is determined. The second step involves the determination of the dynamic contact angle as a function of the applied Ca. This is achieved by linearization of the classical HoffmanTanner law (Bonn et al., 2009). For dynamic systems with non-zero static contact angle, the Hoffman-Tanner law can be slightly modified and extended as (Bertheir, 2008; Khandekar et al., 2010):

$\theta_{d}^{3}-\theta_{s}^{3}=A \cdot \mathrm{Ca}$

where, $\mathrm{A}$ is a constant. If $\mathrm{Ca}$ is small, linearization of Eq. (12) leads to (Berthier, 2008):

$\theta_{d}=\left(\theta_{s}^{3}+A \cdot \mathrm{Ca}\right)^{1 / 3} \approx \theta_{s}\left(1+\frac{1}{3} \frac{A \cdot \mathrm{Ca}}{\theta_{s}^{2}}\right)$

or

$\theta_{d}-\theta_{s} \approx \frac{1}{3} \frac{A \cdot \mathrm{Ca}}{\theta_{s}^{2}}$

It should be noted that $\mathrm{Ca}$ takes the sign of the velocity in the reference frame of the problem. Thus, $\left(\theta_{d}-\theta_{s}\right)$ takes the sign of $\mathrm{Ca}$ and the values of dynamic advancing contact angle is as:

$\theta_{a d v}=\theta_{s}+\frac{1}{3} \frac{A|\mathrm{C} a|}{\theta_{s}^{2}}$

This equation informs us that the advancing contact angle is larger than the static contact angle. As a last step, the dynamic steady state of meniscus motion, at a constant flow velocity, is simulated. Under such conditions, the dynamic contact angle remains constant, as given by Eq. 14.

\section{RESULTS AND DISCUSSION}

Results obtained both experimentally as well as by simulation, are reported hereunder in the following sequence:

(i) Shape of liquid-air meniscus formed inside the tube at different flow Capillary numbers when dynamic steady-state motion of the meniscus is achieved inside the capillary tube,

(ii) Fluid flow field obtained by micro-PIV close to meniscus at different flow Capillary numbers, and,

(iii) Parametric study highlighting the effect of wettability of the liquidtube material combination on the near-meniscus hydrodynamics.

\subsection{Static and dynamic meniscus shape}

The static to dynamic steady-state meniscus shapes are captured by using high speed camera at different Capillary numbers in the square glass capillary. A range of Capillary numbers is obtained by using four different fluids, i.e., water, ethanol, glycerin and silicon-oil $(\rho=760$ $\mathrm{kg} / \mathrm{m}^{3}, \mu=0.0046 \mathrm{~Pa} . \mathrm{s}, \sigma=0.0188 \mathrm{~N} / \mathrm{m}$ ). Inside the dry capillary tube, the static contact angle of water and glycerin was found to be higher than $90^{\circ}\left(101.5^{\circ}\right.$ for water and $107.2^{\circ}$ for glycerin, $)$ and it is less than $90^{\circ}$ for ethanol and silicon oil. Figure 3 clearly depicts that the contact angle of the meniscus drastically changes (static to dynamic) when the applied flow Capillary number is altered. At low Capillary number, the dynamic water-air meniscus remains nearly flat, close to being perpendicular to the channel wall. However, increase in Capillary number results in convexity of the meniscus shape, due partly to the variation of dynamic pressure difference between the two sides of the water-air meniscus. The behavior of glycerin-air meniscus is qualitatively similar to water-air system for the chosen range of Capillary number. For ethanol, the meniscus remains concave in shape for the entire range of applied Capillary numbers. In contrast, for the case of silicon oil, a drastic change of contact angle occurs with respect to the applied Capillary number; the shape of the interface changes from concave (at low $\mathrm{Ca}$ ) to convex (at high $\mathrm{Ca}$ ). These results clearly indicate the strong and unequivocal connection between the wetting characteristics, local interface velocity, and the resulting dynamic pressure, which eventually is intrinsically connected to the local meniscus curvature and thus the resulting shape. It is clear that only Capillary number as a parameter fails to predict the steady-state dynamics shape of the meniscus. It logically follows, as will be demonstrated in the following results that near meniscus transport of momentum (so also, thermal energy in the case of heat transfer systems and mass transport for chemically reacting fluids with the tube wall) will be quite different for these cases.

The contact angle at dynamic steady state is approximately equal to the dynamic contact angle obtained from Eq. 14. In addition, no change in the dynamic contact angle has been observed during the steady motion of meniscus, for glycerin, ethanol and silicon oil. Only for high surface tension system, like water, the steady meniscus motion can show some tendency of intermittent motion (stick-slip tendency) due to local pinning of the three-phase contact line. For a given static contact angle corresponding to the experimental condition, the shape of meniscus at various Capillary numbers has been captured by simulation. A sample result is as shown in Figure 4, which compares the experimental and simulation menisci shapes inside dry-smooth mini-capillary of size $1 \mathrm{~mm} \times 1 \mathrm{~mm}$ at four different flow Capillary numbers. The average difference in the curvature obtained by experiments and simulation is within 11\%; similar results were obtained with the other fluids also. (a) Water

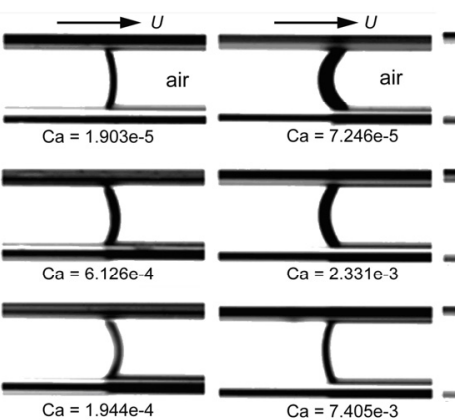

Fig. 3 Experimentally observed variation of meniscus shape with

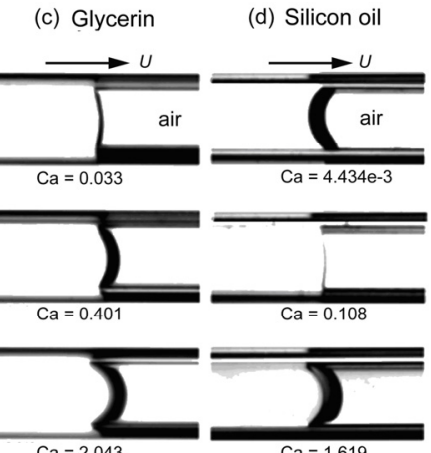
increase in Capillary number for four different fluids: (a) water, (b) ethanol, (c) glycerin, and (d) silicon oil. 
(a) Experimental

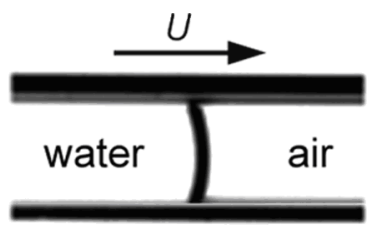

(b) Simulation

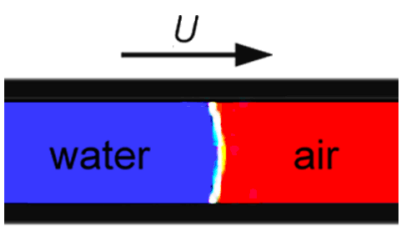

(i) $\mathrm{Ca}=1.903 \mathrm{e}-5$
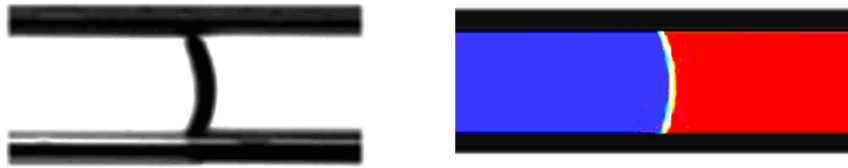

(ii) $\mathrm{Ca}=6.126 \mathrm{e}-4$
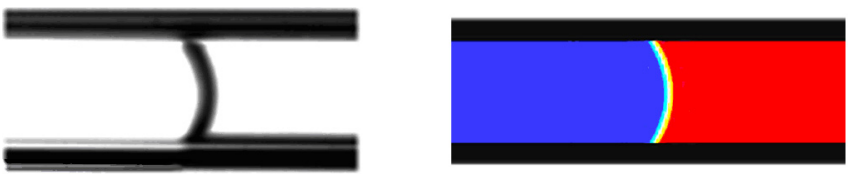

(iii) $\mathrm{Ca}=1.944 \mathrm{e}-4$

Fig. 4 Comparison of meniscus shape of water-air interface using (a) Experimental images of the moving meniscus and, (b) Simulations at corresponding Capillary numbers.

\subsection{Near-meniscus hydrodynamics}

Figure 5 (a) shows a typical image obtained by the PIV objective microscope with the relevant coordinate system. This image has been obtained at the mid-plane $(x-y$ plane) from the top view of the horizontally placed capillary tube (gravity acts in the $z$-direction). Here, the meniscus motion is towards the $x$-direction at $\mathrm{Ca}=2.27 \times 10^{-6}$. Due to the curvature of the meniscus, capturing the seeded particles extremely close to the meniscus is difficult and leads to weak signal for post-processing. Therefore, some finite area just close to the meniscus needs to be masked for the cross-correlation analysis to obtain the flow velocities so that spurious signals are filtered. These have been highlighted in Figure 5(a) for the PIV image. The corresponding image obtained by simulation is shown in Figure 5(b). While there are no such physical restrictions during three-dimensional simulations, as encountered in the experiments, for the sake of one-to-one direct comparison of the two strategies, the corresponding masked area is also shown in Figure 5(b). All experimentally obtained values reported here are typically in the un-masked portions of the flow.

Figure 6 (a) shows the contour of velocity components $(u, v$ and $\partial w / \partial z)$ of liquid moving in the square capillary obtained by microPIV measurements at $\mathrm{Ca}=2.27 \times 10^{-6}$ (working fluid: water) moving inside the square capillary tube. The velocity analysis has been done for the mid-plane (x-y plane) of the capillary tube, as detailed in Figure 4. Figure 6(a) -(i) and (ii), give the experimentally obtained values of velocities $u$ and $v$, respectively, in the directions $\mathrm{x}$ and $\mathrm{y}$. As will be appreciated, micro-PIV analysis only provides two-dimensional $(u, v)$ data at any given plane. Invoking the $2 \mathrm{D}$ continuity equation, the threedimensionality of the flow, if present, can be ascertained - a finite residual of the 2D continuity indicates the presence of $\partial w / \partial z$. This is shown in Figure 6(a) -(iii). The corresponding simulation results for $\mathrm{u}$ and $\mathrm{v}$ velocities are shown in Figure 6(b) -(i) and (ii), respectively. Figure 6 (b) - (iii) shows the simulation results for the velocity w in the third-direction, i.e. the z-direction. Exactly corresponding set of results, but at a higher $U_{\text {avg }}=0.277 \mathrm{~mm} / \mathrm{s}\left(\mathrm{Ca}=3.7 \times 10^{-6}\right)$, are shown in Figure 7 (a) and (b), respectively.

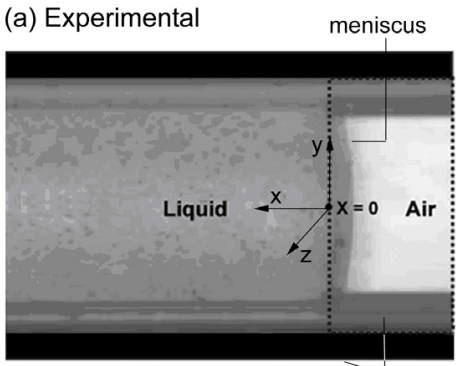

(b) Simulation masked

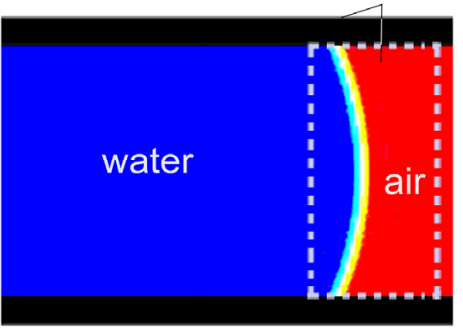

Masked region index

:....... Interface region $\square$ wall region

Fig. 5 (a) View of liquid-air meniscus inside square capillary in the bright field mode. This image was taken at the mid-plane of the channel. The masked region at the interface and wall is defined to remove spurious velocity vectors. The gravity vector points towards the z-direction. (b) Image obtained from simulation, at the same conditions; masked region is marked with dotted line.

From the $u$-contours it is clearly observed that the fluid velocity is higher at the centre of the channel away from the interface and it gradually decreases towards the liquid-air interface. The $v$-contour near to the interface has two dense regions. These regions are having a higher magnitude of $v$-velocity, albeit in opposite directions, as expected due to fluid circulation near the interface. A vortex pair is clearly visible in the v-velocity distribution. As this is a planer 2D data, away from the interface, the mass continuity is nearly satisfied; however, just close the meniscus region the residual error in the $2 \mathrm{D}$ continuity $($ error $=|(\partial U / \partial x)+(\partial V / \partial y)|)$ is more, clearly indicating the existence of gradient of the third component of velocity, $w$. This three-dimensionality in the flow arises due to the drag force on the contact surface with the sidewalls of the micro-channel and the fact that the meniscus must simultaneously obey the free surface boundary condition. These results clearly support the existence of gradient of the third component of velocity, $w$, near the meniscus. The corresponding simulation result shows the actual value of $w$ components near the interface. Experimental data and simulation data for $u$ and $v$ closely match each other. The results also simultaneously establish the threedimensionality of the flow.

To further explore the distribution of the velocity components of the liquid flow near the moving meniscus, Figure 8 and Figure 9 show the variation of velocity components $u$ and $v$ along the channel height at various locations from the liquid-air interface, corresponding to average velocity of $0.166 \mathrm{~mm} / \mathrm{s}\left(\mathrm{Ca}=2.27 \times 10^{-6}\right)$ and $0.277 \mathrm{~mm} / \mathrm{s}(\mathrm{Ca}$ $\left.=3.7 \times 10^{-6}\right)$, respectively. Non-dimensionalization of both $u$ and $v$ velocities has been done using the respective $U_{\text {avg }}$, which is the average liquid supply velocity based on the total cross-section of the channel. Close to the interface a significant drop in the magnitude of the $u$ velocity is seen at the centre region of the channel and this behavior is more prominent at higher flow rates, as can be seen from Figure 8 (a) and 9 (a). 
(a) Experimental
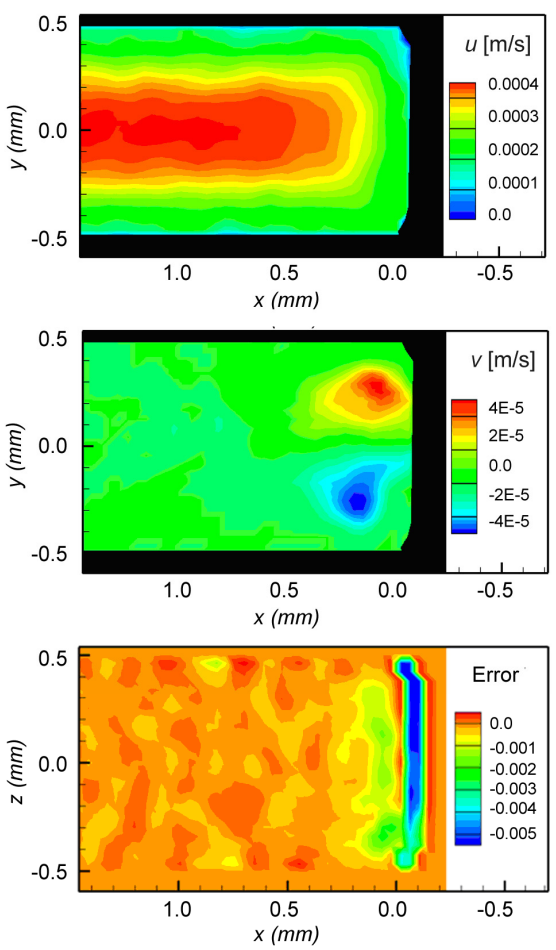

(b) Simulation
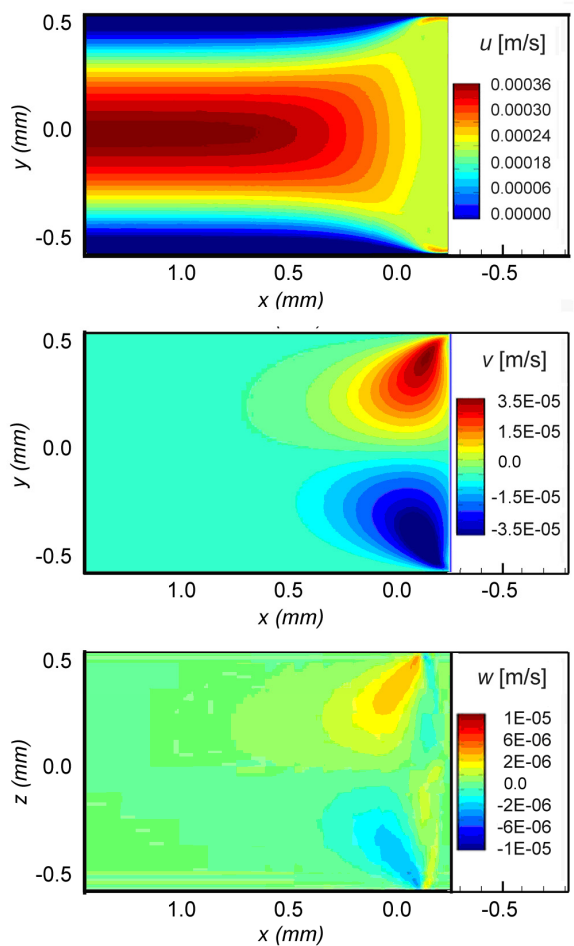

Fig. 6 Flow field at the moving liquid-air interface inside the square-microchannel for $U_{\text {avg }}=0.166 \mathrm{~mm} / \mathrm{s}$. (a) $u, v$ and $\partial w / \partial z$ contour obtained by micro-PIV and (b) $u, v$ and $w$ contour obtained by simulation.

(a) Experimental
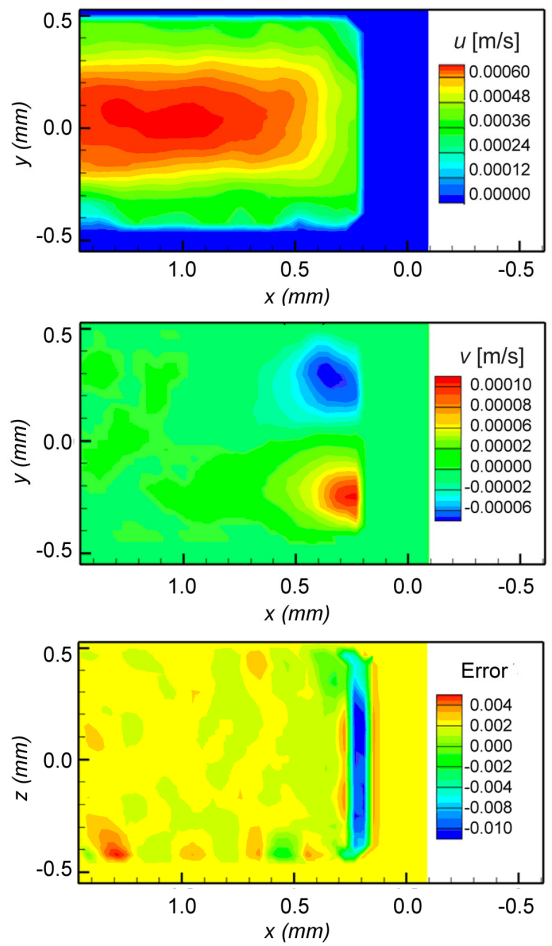

(b) Simulation
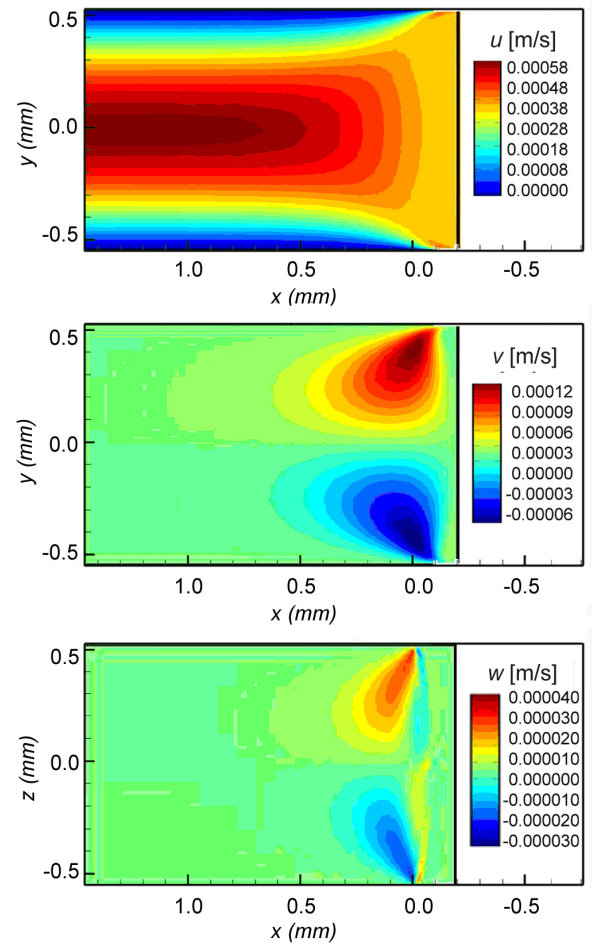

Fig. 7 Flow field at the moving liquid-air interface inside the square-microchannel for $U_{\text {avg }}=0.277 \mathrm{~mm} / \mathrm{s}$. (a) $u, v$ and $\partial w / \partial z$ contour obtained by micro-PIV and (b) $u, v$ and $w$ contour obtained by simulation. 

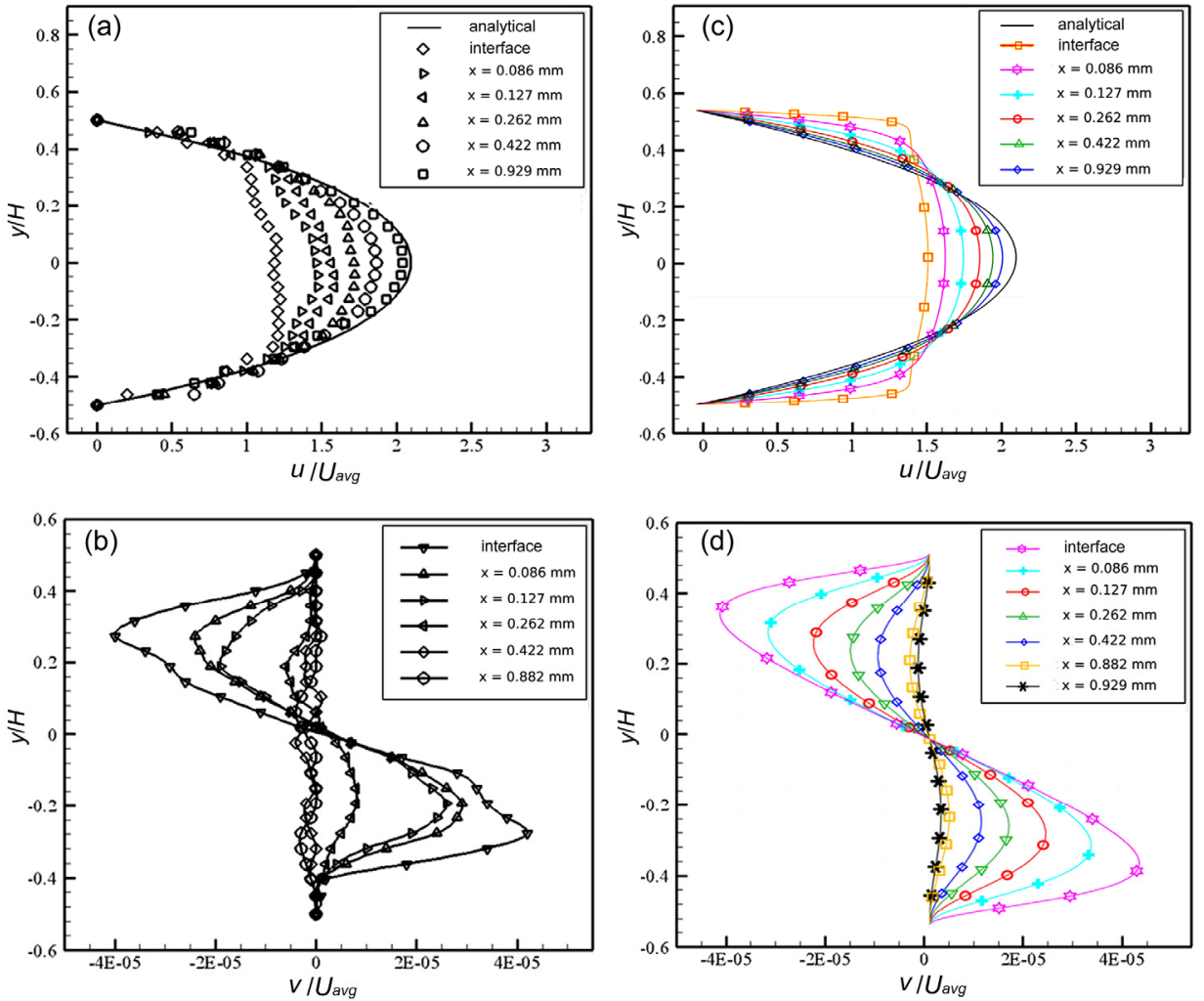

Fig. 8 Variation of non-dimensional $u / U_{\text {avg }}$ and $v / U_{\text {avg }}$ components of velocities with respect to distance from the liquid-vapor interface for average velocity of flow $\left(U_{\text {avg }}\right)$ is $=0.166 \mathrm{~mm} / \mathrm{s}\left(\mathrm{Ca}=2.27 \times 10^{-6}\right)$. (a) and (b) are observed experimentally and (c) and (d) are obtained from simulation, respectively. All the measurements have been performed at the mid-plane of the channel cross-section. $x$ is the streamwise axial distance from the interface ( $x=0$ corresponds to the interface).
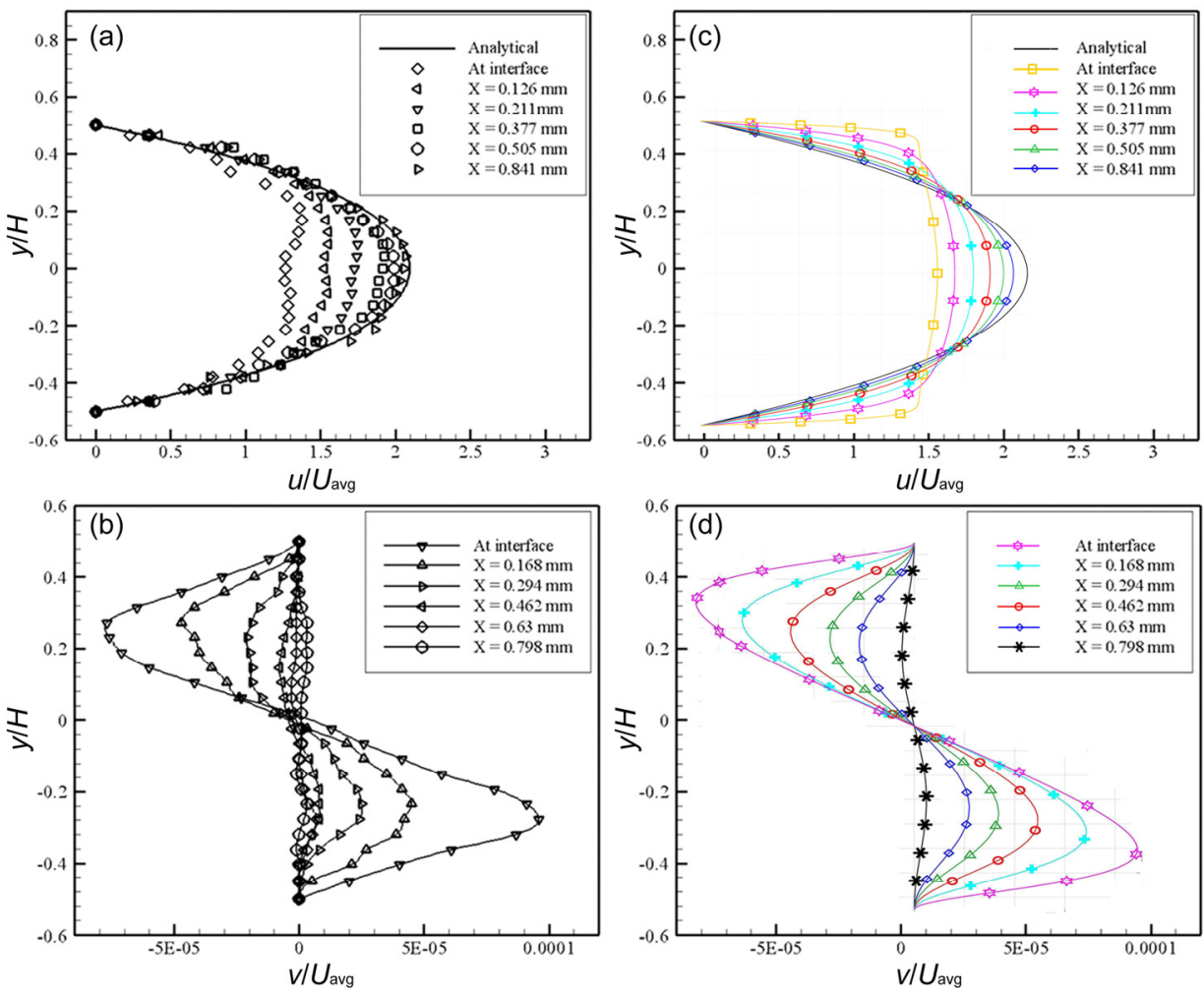

Fig. 9 Variation of non-dimensional $u / U_{\text {avg }}$ and $v / U_{\text {avg }}$ components of velocities with respect to distance from the liquid-vapor interface for average velocity of flow $\left(U_{\text {avg }}\right)$ is $=0.277 \mathrm{~mm} / \mathrm{s}\left(\mathrm{Ca}=3.7 \times 10^{-6}\right)$. (a) and (b) are observed experimentally and (c) and (d) are obtained from simulation, respectively. All the measurements have been performed at the mid-plane of the channel cross-section. $x$ is the streamwise axial distance from the interface $(x=0$ corresponds to the interface). 

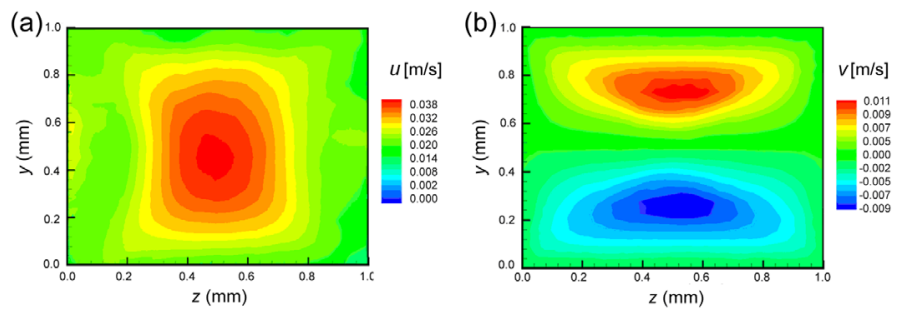

(c)

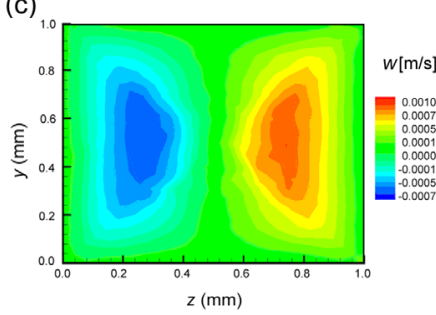

(d)

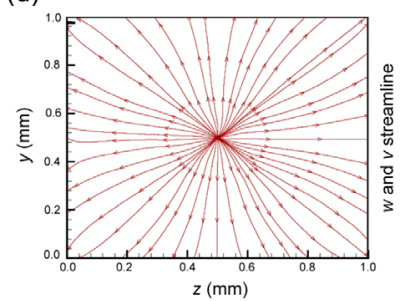

Fig. 10 Liquid flow pattern inside a square capillary tube of size 1.0 $\mathrm{mm} \times 1.0 \mathrm{~mm}$, in the cross-sectional plane $(z-y)$ close to the meniscus, at $x=0.02 \mathrm{~mm}$. The dynamic contact angle is $105^{\circ}$ and applicable flow $\mathrm{Ca}=5 \times 10^{-4}$ (a) contour of $u$-velocity component, (b) contour of $v$-velocity component, (c) contour of w-velocity component, and (d) streamline of $w$ and $v$ velocity components.

This behavior is a direct outcome of the obstruction of the fluid flow at the liquid-air interface, the meniscus giving a free-surface boundary condition with only tangential velocities possible. As we move away from the interface, the u-velocity distribution asymptotically adheres to the parabolic Poiseuille distribution. For benchmarking, the analytical profile, given by Eq. 1 has also been plotted. Figures 8 (b) and 9 (b) show the $v / U_{\text {avg }}$ with respect to height of channel at various points away from the liquid-air interface. Close to interface the $v$-velocity magnitude considerably increases (obviously at the expense of the decrease in the $u$-velocity) and qualitatively varies like a sinusoid. The magnitude of this wave like variation of the $v$ component along the height of channel decreases as the point of observation moves away from the interface. The $v$-velocity eventually becomes negligible away from the interface. As has been pointed out earlier, close to the interface, the flow actually becomes threedimensional in nature, the evidence of which can only be captured indirectly via the residual error in the $2 \mathrm{D}$ continuity, as was shown earlier in Figure 6 (a) -(iii) and Figure 7 (a) -(iii).

Figure 8 (b) and Figure 9(b) show the corresponding simulation results for $u$ and $v$ velocity profiles. The comparison with the experiments suggest that while majority of the flow physics is nicely captured, both qualitatively and quantitatively; however, some minor discrepancies between the simulation and experiments are due primarily because of: (a) the glass capillary tube can have local nonhomogeneity in the wall topography, which leads to local pinning of the three phase contact line, enhancing friction intermittently (b) errors also creep in during the image processing of the PIV data, wherein the data quality close to the wall and just behind the meniscus is limited.

Figure 10 shows the contours of the velocity components $(u, v$ and $w$ ) and the streamline of $w$ and $v$ components in cross section of flow $(z-y)$ at $x=0.02 \mathrm{~mm}$ from the interface (near the water-air interface), for dynamic contact angle $105^{\circ}$ at $\mathrm{Ca}=5 \times 10^{-4}$. The contour of $u, v$ and $w$ in cross-section plane, as shown in figure 10(a-c), clearly depicts circulation of liquid near the interface. Note the order of magnitudes of the $u, v$, and $w$ components, respectively; u-component being an order of magnitude higher. The flatness of the $u$-contour, the near symmetric nature of the vortex-pair circulatory patterns, and the threedimensionality of the flow close to the meniscus is clearly seen from these simulation results. (a) Static contact angle
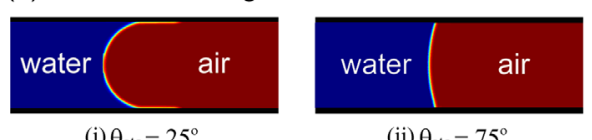

(ii) $\theta_{\mathrm{sta}}=75^{\circ}$

(b) Dynamic contact angle

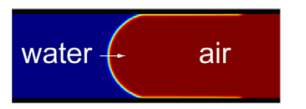

(i) $\theta_{\mathrm{adv}}=25.58^{\circ}$

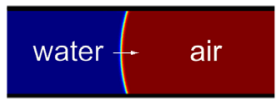

(ii) $\theta_{\text {adv }}=75.05^{\circ}$

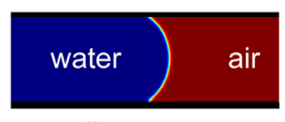

(iii) $\theta_{\mathrm{sta}}=140^{\circ}$

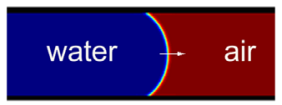

(iii) $\theta_{\text {adv }}=140.01^{\circ}$
Fig. 11 Time evolution of shape of drop change from static contact angle to contact angle at criticality (when the meniscus just commences to move) (a) contact angle $25^{\circ}$, (b) contact angle $70^{\circ}$ and (c) contact angle $140^{\circ}$ for average velocity of flow $\left(U_{\text {avg }}\right)$ is $=0.277 \mathrm{~mm} / \mathrm{s}\left(\mathrm{Ca}=3.7 \times 10^{-6}\right)$.

\subsection{Effect of substrate wettability on flow hydrodynamics}

Having established the simulation procedure and benchmarked against experimental data, one can proceed to study the effect of a range of parameters on the flow. The effect of wettability of the capillary tube (with water) on the hydrodynamics close to the meniscus is presented in this sub-section. Figure 11 shows the time evolution of the shape of meniscus from static contact angle to critical contact angle (the angle at which the meniscus commences its movement) for various wettability scenarios of the capillary tube; the imposed Capillary number is $5 \times 10^{-4}$. For capillary material having high wettability (i.e. static contact angle of $25^{\circ}$, hydrophilic case), the critical contact observed is $25.6^{\circ}$. As the wettability of the capillary tube decreases, the difference between the static contact angle and the critical contact angle diminishes, as can be seen in Figure 11 (a) to (c). Intrinsic wettability also changes the resulting meniscus curvature as can be clearly seen, and this shape directly affects the hydrodynamics of the flow in the wake of the meniscus. Figure 12 (a) shows meniscus shape under dynamic steady state and the resulting flow pattern of fluid close to meniscus inside tubes having a range of wettability (static contact angle for case (i) $25^{\circ}$; case (ii) $70^{\circ}$ and case (iii) $140^{\circ}$ ), at flow $\mathrm{Ca}=5 \times 10^{-4}$. Inside capillary tubes having higher wettability, very thin precursor liquid film layer has been observed close to the three-phase contact line, as shown in Figure 12(a)-i. It is observed that the thickness of this fluid layer decreases as contact angle increases and it approaches zero at a contact angle of $90^{\circ}$. Hydrodynamics of the fluid close to interface has been depicted in Figure 12(b)-(d) for the three cases of different wettability, respectively. Figure 12 (b) represents the streamline of flows while Figures 12 (c) to (e) depicts the velocity contours for $u, v$ and $w$ velocity components respectively, in the $x y$ plane. While all the three cases manifest three-dimensional flow pattern and present a vortex pair, their respective strengths are different. The zone behind the meniscus which gets affected by the vortex flow is a clear function of the wettability of the fluid-tube pair. The fluid perturbation depth is larger for the meniscus formed with a higher wetting fluid. It is clear from these results that the transport of momentum (and so will be the case for heat and mass transport) is a strong function of wetting characteristics. It will directly affect the momentum transport coefficient (skin friction) in the meniscus region, as is explored next.

The variation of Poiseuille number $\left(\mathrm{C}_{\mathrm{f}} \mathrm{Re}\right)$ experienced along the wall due to the steady motion of the meniscus, for the three cases of wettability respectively is shown in Figure 13. Many interesting features can be concluded from this result, in conjunction with Figure 12. Firstly, momentum flux coefficient rapidly increases as we move closer to the three-phase contact line. Higher the wettability of the fluid, higher is the value of $C_{f}$, close to the meniscus. As we move away from the meniscus viscous stress reduces and it becomes asymptotically closer to the Poiseuille flow situation. 

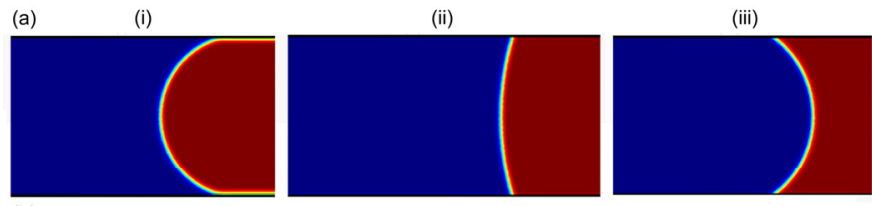

(b)
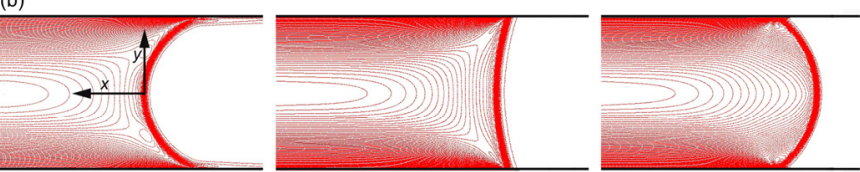

(c)
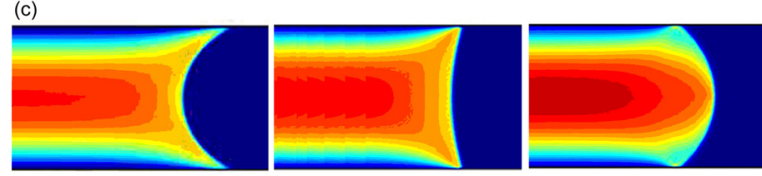

(d)
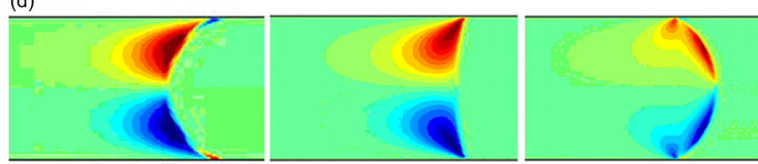

(e)
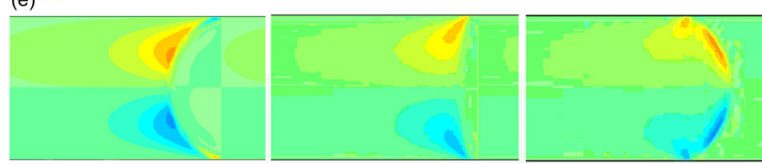

Fig. 12 (a) Meniscus shape for various wettability capillary tube at commencement of motion, (b) $u-v$ streamline in $x-y$ plane, (c) contour of $u$ components of velocity in $x-y$ plane, (d) contour of $v$ components of velocity in the $x-y$ plane and (e) contour of $w$ components of velocity in $x-z$ plane for various wettability of capillary tube, (i) contact angle $25^{\circ}$, (ii) contact angle $70^{\circ}$ and (iii) contact angle $140^{\circ}$ for average velocity of flow $\left(U_{\text {avg }}\right)$ is $=0.038 \mathrm{~mm} / \mathrm{s}\left(\mathrm{Ca}=5 \times 10^{-4}\right)$. (Note: All data representation is in the moving frame of reference)

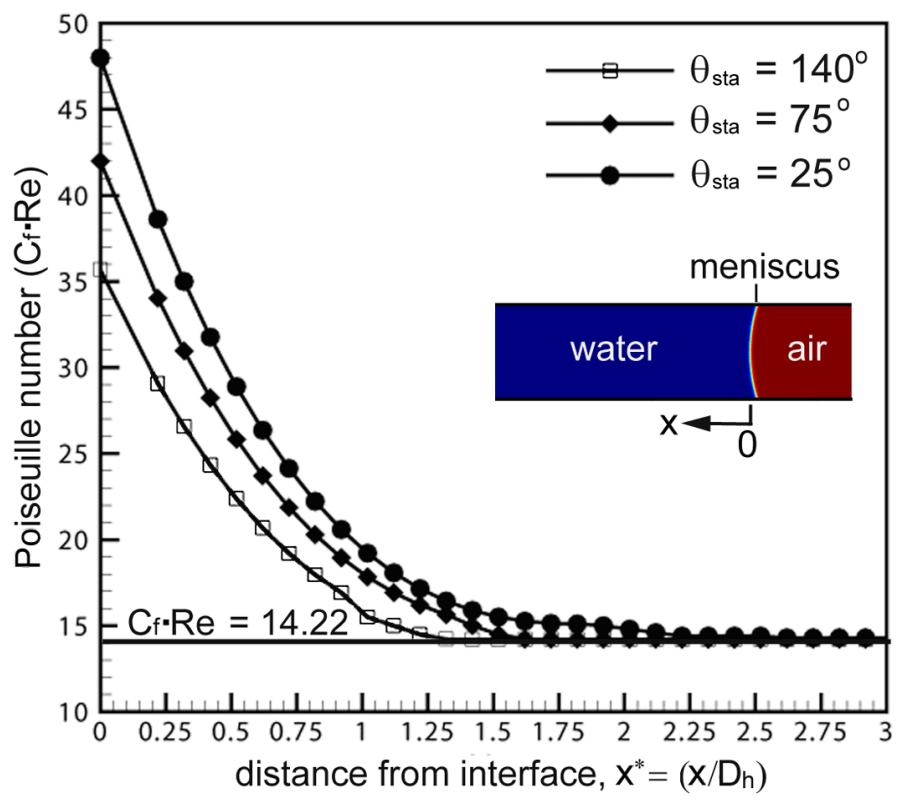

Fig. 13 Variation of Poiseuille number $\left(\mathrm{C}_{\mathrm{f}} \mathrm{Re}\right)$ experienced along the wall due to the steady motion of the meniscus, for the three cases of wettability respectively.
For reference, the skin friction coefficient for fully developed singlephase liquid flow in a rectangular channel, i.e., $\mathrm{C}_{\mathrm{f}}=14.22$, is also shown on the figure. The effect of the meniscus is felt at a distance of about $2 \mathrm{D}_{\mathrm{h}}$ to $3 \mathrm{D}_{\mathrm{h}}$, this distance being a function of the wettability too. Since a small contact angle is related to a large dispersion force, a stronger dispersion force enhances the interaction between liquid phase and solid substrate and improves the wetting (Churaev 1995). With similar surface roughness of the micro-channels, the surface energy dissipation of the hydrophobic micro-channel is lower, while the interaction at the liquid-solid interface is weaker. Significant drag reduction has also been reported using hydrophobic surfaces in many studies (Tretheway and Meinhart 2002; Choi et al. 2003).

\section{SUMMARY AND CONCLUSIONS}

In the present work, dynamic shape of an isolated moving meniscus formed between a liquid-air system inside a square mini-channel has been studied for water, ethanol, glycerin and silicon-oil, with varying $\mathrm{Ca}$. It is shown that a drastic change in the dynamic shape of the meniscus can occur due to the imposed bulk velocity. Subsequently, using micro-PIV, liquid velocity distributions near the moving water-air meniscus were measured at the mid-plane of the channel, at low $\mathrm{Ca}$. It was shown that near the liquid-air interface the non-dimensional $u$ velocity profiles are flatter in nature, while moving away from the meniscus the profiles follow the fully developed flow profile. The $v$ velocity is moderately dominant only close to the interface, although its absolute value is rather small as compared to the $u$-velocity. Formation of opposite vortices is clearly indicated by the $v$-profiles. Checking the continuity close to the interface indicated that the flow is indeed threedimensional in nature in the proximity of the interface. It is highlighted that near meniscus local transport phenomena are critical in understanding the transport behavior of micro-scale multi-phase systems. The experimental data for the flow field close to moving liquid-air interface are compared with numerical simulations. After benchmarking, the wettability parameter is varied in the simulations to highlight its isolated effect on local flow transport characteristics. The results clearly demonstrate that, close to the meniscus, the flow clearly becomes three-dimensional with both $v$ and $w$ velocities showing a double-vortex, the strength of the latter being an order-of-magnitude lower than the former. This three-dimensionality leads to enhanced local transport. The simulations also reveal that controlling the wettability of the liquid can be an effective tool to control the viscous shear; the meniscus region of wetting liquids manifest stronger shear stress as compared to a non-wetting liquid moving at the same Capillary number. It is expected that the heat and mass transfer near the meniscus region will also be qualitatively affected in a similar fashion as the momentum flux transfer, taking the cue from Prandtl and Schmidt number analogy. Thus, it is concluded from this study that wettability of the fluid is an important parameter for species transfer under dynamic conditions near the meniscus regions in microscale geometries.

\section{ACKNOWLEDGEMENTS}

Financial support from the Indo-French Centre for the Promotion of Advanced Research (IFCPAR/CEFIPRA), New Delhi (Project \#: 44081/2010), under the aegis of Indian Department of Science and Technology and the French Ministry of Foreign Affairs is gratefully acknowledged. 


\section{NOMENCLATURE}

$\begin{array}{ll}A & \text { constant }(-) \\ \mathrm{Ca} & \text { Capillary number, }\left(\mu U_{\text {avg }} / \sigma\right) \\ \mathrm{C}_{\mathrm{f}} & \text { skin friction coefficient }(-) \\ \mathrm{Co} & \text { Courant number }\left(\Delta t /\left(\Delta x /\left(U_{\text {avg }}\right)_{l}\right)\right) \\ F & \text { force density }\left(\mathrm{N} / \mathrm{m}^{3}\right) \\ h & \text { height }(\mathrm{m}) \\ Q & \text { flow rate }\left(\mathrm{m}^{3} / \mathrm{s}\right) \\ n & \text { unit normal vector }(-) \\ \mathrm{Po} & \text { Poiseuille number }\left(\mathrm{C}_{\mathrm{f}} \cdot \operatorname{Re}\right) . \\ \operatorname{Re} & \text { Reynolds number }\left(\rho U_{\text {avg }} D / \mu\right) \\ U & \text { velocity }(\mathrm{m} / \mathrm{s}) \\ u & \text { X-component of velocity }(\mathrm{m} / \mathrm{s}) \\ v & \text { y-component of velocity }(\mathrm{m} / \mathrm{s}) \\ w & \text { Z-component of velocity }(\mathrm{m} / \mathrm{s}) \\ x, y, z & \text { directions in Cartesian coordinates }(-)\end{array}$

Greek symbols

$\begin{array}{ll}\phi & \text { level-set parameter (-) } \\ \delta & \text { dirac-delta function (-) } \\ \mu & \text { dynamic viscosity (Pa-s) } \\ \kappa & \text { radius of interface curvature (m) } \\ \rho & \text { density }\left(\mathrm{kg} / \mathrm{m}^{3}\right) \\ \sigma & \text { surface tension }(\mathrm{N} / \mathrm{m}) \\ \theta & \text { contact angle (deg or rad) } \\ \xi & \text { non-dimensional distance }(-)\end{array}$

\section{Subscripts}

adv advancing

air air/gas-phase

avg average

$h \quad$ hydraulic

$l \quad$ liquid

d dynamic

$s \quad$ static, surface

$g \quad$ gravitational, volumetric

\section{REFERENCES}

Ajaev, V.S., and Homsy, G.M., 2006 "Modelling Shapes and Dynamics of Confined Bubbles," Annual Review of Fluid Mechanics, 38, 277-307. http://dx.doi.org/10.1146/annurev.fluid.38.050304.092033

Bajpai, A.K., and Khandekar, S., 2012, "Thermal Transport Behavior of a Liquid Plug Moving Inside a Dry Capillary Tube," Heat Pipe Science and Technology, 3, 97-124.

http://dx.doi.org/10.1615/HeatPipeScieTech.2013006563

Berthier, J., 2008, Microdrops and Digital Microfluidics. William Andrew Inc., New York, USA.

Blake, T.D., 2006, "The Physics of Moving Wetting Lines," Journal of Colloids and Interface Science, 299, 1-13.

http://dx.doi.org/10.1016/j.jcis.2006.03.051

Bonn, D., Eggers, J., Indekeu, J., Meunier, J., and Rolley, E., 2009, "Wetting and Spreading," Review of Modern Physics, 81,739-805.

http://dx.doi.org/10.1103/RevModPhys.81.739

Brackbill, J.U., Kothe, D.B., and Zemach, C., 1992, “A Continuum Method for Modeling Surface Tension," Journal of Computational Physics, 100(2), 335-354.

http://dx.doi.org/10.1016/0021-9991(92)90240-Y

Bruus, H., 2008, Theoretical Microfluidics. Oxford Master Series in Physics, ISBN-13: 978-0199235087, Oxford University Press, New York, 48-52.
Chebbi, R., 2003, "Deformation of Gas-Liquid Interfaces in Capillary Tubes," Journal of Colloid and Interface Science, 265, 166-173.

http://dx.doi.org/10.1016/S0021-9797(03)00415-6

Choi, C.H, Westin, K.J.A, and Breuer, K.S., 2003, “Apparent Slip Flows in Hydrophilic and Hydrophobic Micro-Channels," Physics of Fluids, 15(10), 2897-2902.

http://dx.doi.org/10.1063/1.1605425

Churaev, N.V., 1995, "Contact Angles and Surface Forces," Advances in Colloid and Interface Science, 58(2-3), 87-118.

http://dx.doi.org/10.1016/0001-8686(95)00245-L

Egorov, A.G., Kornev, K.G., and Neimark, A.V., 2003, "Meniscus Motion in a Prewetted Capillary," Physics of Fluids, 15, 3134-3143.

http://dx.doi.org/10.1063/1.1604392

Gunther, A., Khan, S.A., Thalmann, M., Trachsel, F., and Jensen, K.F., 2004, "Transport and Reaction in Microscale Segmented Gas-Liquid Flow," Lab on Chip, 4, 278-286. http://dx.doi.org/10.1039/B403982C

He, Q., Hasegawa, Y., and Kasagi, N., 2010, "Heat Transfer Modeling of Gas-Liquid Slug Flow without Phase Change in a Micro-Tube," International Journal of Heat and Fluid Flow, 31, 126-136. http://dx.doi.org/10.1016/j.ijheatfluidflow.2009.11.004

Hoffman, R.L., 1975, “A Study of Advancing Interface,” Journal of Colloid and Interface Science, 50, 228-241. http://dx.doi.org/10.1016/0021-9797(75)90225-8

Huh, D., Kuo, C-H., Grotberg, J.B., and Takayama1, S., 2009, "Gas-Liquid Two-Phase Flow Patterns in Rectangular Polymeric Microchannels, Effect of Surface Wetting Properties," New Journal of Physics, 11, 075034(14). http://dx.doi.org/10.1088/1367-2630/11/7/075034

Juncker, D., Schmid, H., Drechsler, U., Wolf, H., Michel, B., Rooij, N.D., and Delamarche, E., 2002, "Autonomous Microfluidic Capillary System," Analytical Chemistry, 74(24), 6139-6144.

http://dx.doi.org/10.1021/ac0261449

Khandekar, S., Panigrahi, P.K., Lefevre, F., and Bonjour, J., 2010, "Local Hydrodynamics of Flow in a Pulsating Heat Pipe: A Review," Frontiers in Heat Pipes, 1, 1-20.

http://dx.doi.org/10.5098/fhp.v1.2.3003

King, C., Walsh, E., and Grimes, R., 2007, "PIV Measurement of Flow within Plugs in a Microchannel," Microfluidics Nanofluids, 3, 463-472. http://dx.doi.org/10.1007/s10404-006-0139-y

Kinoshita, H, Kaneda, S, Fujii, T, and Oshima, M, 2007, "ThreeDimensional Measurement and Visualization of Internal Flow of a Moving Droplet Using Confocal Micro-PIV," Lab on Chip, 7, 338-346. http://dx.doi.org/10.1039/b617391h

Lee, C.Y., and Lee, S.Y., 2008, "Pressure Drop of Two-Phase Plug Flow in Round Mini-Channels: Influence of Surface Wettability," Experimental Thermal and Fluid Science," 32(8), 1716-1722.

http://dx.doi.org/10.1016/j.expthermflusci.2008.06.007

Li, H., and Olsen, M., 2006, "Micro-PIV Measurement of Turbulent Flow in Square Microchannels with Hydraulic Diameters from $200 \mu \mathrm{m}$ to 640 $\mu \mathrm{m}, "$ International Journal of Heat and Fluid Flow, 27, 123-134. http://dx.doi.org/10.1016/j.ijheatfluidflow.2005.02.003

Lima, R., Wada, S., Takeda, M., Tsubota, K., and Yamaguchi T., 2007, "In vitro Confocal Micro-PIV Measurements of Blood Flow in a Square Microchannel: the Effect of the Haematocrit on Instantaneous Velocity Profiles," Journal of Biomechanics, 40, 2752-2757. http://dx.doi.org/10.1016/j.jbiomech.2007.01.012

Malsch, D., Kielpinski, M., Merthan, R., Albert, J., Mayer, G., Kohler, M., Stahl, M., and Henkel, T., 2008, "Micro-PIV Analysis of Taylor Flow in Microchannels," Chemical Engineering Journal, 135S, 166-172. http://dx.doi.org/10.1016/j.cej.2007.07.065 
Mehdizadeh, A., Sherif, S.A., and Lear, W.E., 2011, "Numerical Simulation of Thermofluid Characteristics of Two-Phase Slug Flow in Microchannels," International Journal of Heat and Mass Transfer, 54, 3457-3465. http://dx.doi.org/10.1016/i.ijheatmasstransfer.2011.03.040

Meinhart, C.D., Wereley, S.T., and Santiago, J.G., 1999, "PIV Measurement of a Microchannel Flow," Experiments in Fluids, 27, 414-419. http://dx.doi.org/10.1007/s003480050366

Olsson, E., and Kreiss, G., 2005, "A Conservative Level-Set Method for Two-Phase Flow," Journal of Computational Physics, 210, 225-246. http://dx.doi.org/10.1016/j.jcp.2005.04.007

Pattekar, A.V., and Kothare, M.V., 2004, "A Microreactor for Hydrogen Production in Micro Fuel Cell Applications," Journal of Micro-electroMechanical Systems, 13, 7-18. http://dx.doi.org/10.1109/JMEMS.2004.823224

Rose, W., and Heins, R.W., 1962, "Moving Interfaces and Contact Angle Rate-Dependence," Journal of Colloid and Interface Science, 17, 39-48. http://dx.doi.org/10.1016/0095-8522(62)90074-0

Santiago, J.G., Wereley, S.T., Meinhart, C.D., Beebe, D.J., and Adrian, R.J., 1998, "A Particle Image Velocimetry System for Microfluidics," Experiments in Fluids, 25, 316-319. http://dx.doi.org/10.1007/s003480050235

Silva, G., Leal, N., and Semiao, V., 2008, "Micro-PIV and CFD Characterization of Flows in a Microchannel: Velocity Profiles, Surface Roughness and Poiseuille Numbers," International Journal of Heat and Fluid Flow, 29, 1211-1220.

http://dx.doi.org/10.1016/j.ijheatfluidflow.2008.03.013

Sussman, M., Fatemi, E., Smereka, P., and Osher, S., 1998, “An Improved Level-Set Method for Incompressible Two-Phase Flows," Computers \& Fluids, 27, 663-680.

http://dx.doi.org/10.1016/S0045-7930(97)00053-4

Sussman, M., Smereka, P., and Osher, S., 1994, “A Level-Set Approach for Computing Solutions to Incompressible Two-Phase Flow," Journal of Computational Physics, 114, 146-159.

http://dx.doi.org/10.1006/jeph.1994.1155

Tanner, L.H., 1979, "The Spreading of Silicone Oil Drops on Horizontal Surfaces," Journal of Physics-D Applied Physics, 12, 1473-1484.

http://dx.doi.org/10.1088/0022-3727/12/9/009
Shah, R.K., and London, A.L., 1978, Laminar Flow Forced Convection in ducts. Academic Press, New York, 1978.

Thulasidas, T.C., Abraham, M.A., and Cerro, R.L., 1997, "Flow Patterns in Liquid Slugs During Bubble Train Flow Inside Capillaries," Chemical Engineering Science, 52, 2947-2962.

http://dx.doi.org/10.1016/S0009-2509(97)00114-0

Tornberg, A.K., and Engquist, B., 2000, "A Finite Element Based Level-Set Method for Multiphase Flow Applications," Computing and Visualization in Science, 3, 93-101.

http://dx.doi.org/10.1007/s007910050056

Torralba, M., Pita, J.R., Pita, A.A., Huelsz, G., Rio, A.D., and Ortin, J., 2005, "Measurement of the Bulk and Interfacial Velocity Profiles in Oscillating Newtonian and Maxwellian Fluids," Physical Review-E, 72, 0163081-0163089.

http://dx.doi.org/10.1103/PhysRevE.72.016308

Tretheway, D.C., and Meinhart, C.D., 2002, "Apparent Fluid Slip at Hydrophobic Microchannel Walls," Physics of Fluids, 14, L9-L12. http://dx.doi.org/10.1063/1.1432696

Walker, G.M., and Beebe, D.J., 2002, "A Passive Pumping Method for Microfluidic Devices," Lab on Chip, 2(3), 131-134.

http://dx.doi.org/10.1039/b204381e

Wang, X., Yong, Y., Yang, C., Mao, Z.S., and Li, D., 2013, “Investigation on Pressure Drop Characteristic and Mass Transfer Performance of GasLiquid Flow in Micro-Channels" Microfluidics and Nanofluidics, 16(1-2), 413-423. http://dx.doi.org/10.1007/s10404-013-1226-5

Yamada, T., Hong, C., Gregory, O.J., and Faghri, M., 2011, "Experimental Investigations of Liquid Flow in Rib-Patterned Microchannels with Different Surface Wettability, Microfluidics and Nanofluidics, 11, 45-55. http://dx.doi.org/10.1007/s10404-011-0771-z

Zimmerman, J.D., Weislogel, M.M., and Tretheway, D.C., 2010, "MicroPIV Measurements Near a Moving Contact Line," in Proc. of ASME International Mechanical Engineering Congress and Exposition (IMECE), Vancouver, Canada, 1295-1297.

http://dx.doi.org/10.1115/IMECE2010-39092 